\title{
Applications of Image Processing Technique in Porous Material Characterization
}

\author{
Ming Gan ${ }^{1}$ and Jianhua Wang 2 \\ ${ }^{1}$ School of Aeronautics \& Astronautics Engineering, Purdue University, IN, \\ ${ }^{2}$ Department of Thermal Science \& Energy Engineering, \\ University of Science \& Technology of China, Hefei, \\ 1USA \\ ${ }^{2} P . R$. China
}

\section{Introduction}

Nondestructive testing (NDT) provides safe operation to engineering components - it eliminates the risk of damage during operation, and does not require specific sample preparation. It has been widely used to detect and evaluate defects, or measure properties of different types of materials and engineering structures. Examples of NDT techniques include ultrasonic, radiography, infrared thermography, electromagnetic techniques and visible optical methods. The imaging principles and imaging facilities used by these techniques can be very different, but almost all the techniques listed above require image processing to some extent. In this chapter, instead of extensively exploring all the NDT techniques and corresponding image processing methods, we will focus on optical measurement technique and related image processing methods, and use porous materials as specimens.

Optical microscopy and digital camera imaging are two examples of optical measurement technique, where optical microscopy is the conventional one. Based on how the light transfers from the sample to the objective, it can be categorized to two different modes transparent mode and reflected mode. Digital camera imaging appeared with the development of semiconductor industry. Digital images bring convenience for image storage, image transferring, and subsequent image processing. Actually, digital image acquisition facilities, e.g. Complementary Metal Oxide Semiconductors (CMOS) and Charge Coupled Device (CCD), can be combined with the traditional microscope to form digital microscopes. As a convenient way of measurement, optical microscope and digital camera imaging system has been extensively used in the area of microelectronics, nanophysics, biotechnology, pharmaceutical research, mineralogy, and material science.

Typical image processing procedures include image acquisition, image alignment/stitching, image contrast enhancement, grey scale thresholding, and/or image subtraction. The characteristic of the specimens determines which image processing techniques to be used. For example, for the images with background noise, image subtraction can be applied when the background can be evaluated; otherwise thresholding might be applied to eliminate the background influence. 
In this chapter, the image processing techniques will be reviewed. Their applications in characterizing porous materials will be explored.

\section{Overview of image processing techniques}

\subsection{Image acquisition}

Image acquisition is the first step of optical characterization techniques. It is also the first place where noise can be introduced. The characteristic of the noise introduced in the image acquisition process dominates the following image processing techniques. In order to understand the cause of noise, the principles of image acquisition devices will be discussed first.

For digital image acquisition, CMOS and CCD are the most commonly used devices. The first solid-state imager presented in the 1960's. The CMOS image sensors appeared in 1967 [1], and the first CCD sensor appeared in 1970 [1]. In early 1970's MOS diodes were used as light sensitive elements [2]. However, at that time, CMOS image sensors had poor performance and large pixel size, compared to CCD. CCD has been the major imaging device owing to their superior dynamic range, lower fixed-pattern noise, higher fill factor, and higher sensitivity to light [3]. However, during the last decade, CMOS sensor implementing buffer per pixel has been developed, which is known as active pixel sensors (APS). APS provides high speed readout, which makes it suitable for high speed imaging [4]. Table 1 is the comparison of CCD and CMOS image sensors.

\begin{tabular}{|c|l|l|}
\hline Features & \multicolumn{1}{|c|}{ CCD } & \multicolumn{1}{c|}{ CMOS } \\
\hline Sensitivity & $\begin{array}{l}\text { Higher, especially for still } \\
\text { images }\end{array}$ & Lower \\
\hline Noise level & Lower & Higher \\
\hline Dark current & Lower & $\begin{array}{l}\text { Magnitudes higher than the } \\
\text { CCD [5] }\end{array}$ \\
\hline High speed performance & - & Good \\
\hline Power consumption & Higher & Lower \\
\hline Compatibility & - & $\begin{array}{l}\text { Higher integration } \\
\text { capability with chip circuits }\end{array}$ \\
\hline
\end{tabular}

Table 1. Comparison of CCD and CMOS

Right now, CMOS is being used in the area of security surveillance, automotive, imaging phones, etc., owing to the properties of lower power consumption, lower operation voltage, high compatibility and high speed readout rate. CCD has application in the area of medical imaging, astronomy, professional cameras, etc., because of its high performance and image quality [6].

In the material characterization area, CCD is the dominating digital imager. CCD device consists of arrays of capacitors, which accumulate electric charge proportional to the light intensity at the location. In an ideal case, every photon striking the CCD sensor will be converted to one electron. Then the intensity of the incoming light can be quantified by 
counting the corresponding electrons. Unfortunately, noise always exists in this process. Noise in CCD images appears in multiple ways, including dark background areas, faint horizontal or vertical lines, blotchy gradients, and low contrast images. A brief overview of noise sources in CCD is listed below.

\section{Dark current}

All CCD sensors generate a dark signal to some degree. CCD builds up dark current whether the CCD is being exposed to light or not. Dark current arises from thermal energy within the silicon lattice in the CCD [7]. Electrons dependent of the incoming light are collected over time by the CCD potential wells and counted as signal electrons. This signal also carries a statistical fluctuation known as dark current noise. The rate of dark current accumulation depends on the temperature of the CCD, but will eventually fill every pixel in a CCD. The pixels in the CCD will be cleared and reset before imaging. However, dark current accumulates again when exposure starts. In the case of long integration time usage of CCD systems, e.g. in astrophotography and spectroscopy, more input signal is collected, as well as more dark current noise. Ideally, the dark current noise should be reduced to a point where its contribution is negligible over a typical exposure time. The rate of dark current can be reduced by a factor of 100 or more by cooling the CCD [7]. CCDs can be cooled either with thermoelectric coolers (TECs) or liquid nitrogen. The amount of dark current noise highly depends on the temperature, with half value of dark current for every $5^{\circ} \mathrm{C} \sim 9{ }^{\circ} \mathrm{C}$ cooling down of the system [8]. Therefore, cooling system is integrated to high sensitivity CCDs, e.g. the CCD used in spectroscopy area, to increase the signal-to-noise ratio. However, running the $\mathrm{CCD}$ cooler than necessary can also lead to extra noise, which appears as a 'ghost' image.

Besides eliminating the dark noise in the hardware level, methodological ways are also necessary. In intensity-oriented measurements, in order to further eliminate the effect of dark signals, sub-exposure of dark frames is usually applied for calibration of the image acquisition system. When acquiring the dark frames, all the other conditions are kept the same. The acquired dark frame is the background of the measurement, which will be subtracted from the measurement images in the following process.

\section{Pixel non-uniformity}

CCD pixels are made to be uniform. Unfortunately, in really practice, they are slightly different to each other. The sensitivity to light of the pixels is typically within $1 \%$ to $2 \%$ fluctuation of the average. This non-uniform sensitivity brings error to the image. Pixel nonuniformity can be reduced by calibration with a flat-field image [9].

One way to take account the dark current noise and pixel non-uniformity is to normalize them using the following equation (Eqn. 2.1) [10].

$$
I_{n}=\frac{I_{o}-I_{\text {dark }}}{I_{\text {flat }}-I_{\text {dark }}}
$$

where $I_{o}$ is the acquired image; $I_{\text {dark }}$ is the dark current frame; $I_{\text {flat }}$ is the flat-field image; $I_{n}$ is the normalized image. 


\section{Shot noise}

The random arrival of photons to the CCD surface introduces shot noise. The probability of the photon arrival follows Poisson distribution. Shot noise becomes obvious when collecting a small amount of photons. Shot noise can be eliminated by collecting more photons, either with a longer exposure time or by combining multiple frames [9].

\subsection{Image alignment/stitching}

Image alignment discovers correspondence among images with a certain degree of overlap. It is essential for image stabilization in computer vision. In material characterization area, it is required when comparing images of the same place of interest, but taken with different shoots. It is the former step of image subtraction. Image alignment techniques include pixelbased alignment [11], feature-based alignment [12-14], Fourier-based alignment [15, 16] and incremental refinement $[17,18]$.

Image stitching combines multiple images of different areas with overlapping to create a panorama. It is useful for obtaining high resolution overview of an object from multiple microscopic images. Image stitching techniques include key point detection $[19,20]$, feature matching [21], geometric registration [22, 23] and global registration [24, 25]. In material characterization area, feature matching is the commonly applied technique. Detailed description of image alignment algorithms and image stitching methods can be found in the technical report written by R. Szeliski [26].

\subsection{Image contrast enhancement}

Electronically acquired images often have grey scale distortion and require contrast enhancement to restore their quality. Image contrast enhancement makes it easier for object detection, edge detection, and image segmentation. However, this technique is only applied when image intensity is not related to the parameter to be measured. The enhancement algorithm is highly affected by the properties of material to be measured. The contrast enhancement algorithms can be classified as histogram-based [27-29] or frequency domainbased [30-32]. Among these algorithms, histogram equalization is the basis of many derivatives. It includes block-based histogram equalization methods such as contrast limited adaptive histogram equalization [27-30, 32-34]. Traditional histogram equalization modifies the histogram of the entire image to obtain a contrast-enhanced image with a more uniform histogram. Although it enhances the contrast to a large extent to produce a better visualization effect, it still cannot discriminate details in homogeneous regions in the image. Chang et al proposed a block-based histogram equalization method, called collaborative learning method for image enhancement, which works well for images with homogeneous regions [35].

\subsection{Thresholding}

Thresholding is one of the traditional image processing methods. It is also the simplest method of image segmentation. In the thresholding process, individual pixels are treated as object pixels if their values are greater or smaller than a threshold value. Thresholding a grey image results in a binary image, which can be used to perform measurement of 
interest, e.g. area fraction, and spatial distribution. Choosing threshold value is essential in image processing. In fraction measurement or porosity detection, slightly different threshold values can lead to dramatic difference in the final results. In practice, the threshold value is affected by measuring conditions also, e.g. illuminatiing intensity.

The thresholding algorithm can be categorized to four groups - histogram shape-based methods [36, 37], entropy-based methods [38], locally adaptive methods [39, 40] and spatial methods[41]. In the histogram shape-based methods, the aim is to find an optimal threshold value that separates two major peaks in the histogram. This is performed by applying smoothing filters to the histogram, followed by applying a difference filter or fitting the histogram. The drawback of the histogram shape-based method is the lack of spatial information of images. The entropy-based methods use the entropy of the image as a constraint for selecting thresholds. There are two ways to conduct this process - maximizing the entropy of the thresholded image or minimizing the cross entropy between input image and the output image [42]. The disadvantage of this method is the complexity and low image quality. Locally adaptive thresholding method calculates threshold at every pixel. The threshold value is determined by local parameters, e.g. mean, variance, surface fitting parameters or their combinations. Due to the algorithm of this method, the calculation of threshold is usually time-consuming. Spatial methods use higher-order probability distribution and/or correlation between pixels for thresholding.

Recently, wavelet transformation fuzzy set theory [43], and Parzen window estimate [44] technique are applied to create multi-level thresholing methods. More details on image thresholding methods can be found in the article written by Sezgin and Sankur [45].

\subsection{Subtraction}

Image subtraction is the process in which one reference/background image is 'subtracted' from measurement images, to record changes in luminosity or to remove the effect of the background. In the case of subtracting reference image, the images were focused on the same measurement place, but taken at different time slots. The change in luminosity may be from object movement or property change of the area to be measured. It is applicable in the area of particle image velocimetry, star movement detection in astronomy, angiography of blood vessels and porosity detection of porous materials. The key point of reference image subtraction technique is to create localized luminosity change of particles or areas to be measured, while keeping the luminosity of the other areas with minimum change. The methods to illuminate the particles or areas include visible light illumination, laser (UV, visible, IR) illumination, and fluorescence effect. In the case of star movement detection, no artificial illumination needed. However, the objects to be measured usually do not possess such optical properties. Therefore, assistant illumination additives are applied to reveal the properties of interest. In particle image velocimetry, seeding particles are added to the fluid and illuminated with external light source; in angiography of blood vessels, fluorescence effect is applied to reveal the blood flow; in porosity detection of porous materials, purified water is used to reveal the location of penetrating pores by reflecting visible light. The reference images are taken at the same conditions but before the property (e.g. position, velocity) changes. In order to keep the conditions unchanged during taking reference and measurement images, vibration control, temperature control, and shooting parameter 
control are necessary. To further maintain consistency between different images, calibration of dark noise, image alignment, image thresholding techniques would also be useful.

\subsection{3-D reconstitution}

3-D reconstitution of images usually requires several image processing steps. It includes image reconstruction, image enhancement, image classification, and structure clustering. The techniques involved here are similar to the ones we discussed previously, but with focus on 3-D image processing.

3-D images are obtained by stacking sequence of 2-D slice images, which are tomographic reconstructions from projection images. The commonly used algorithm for image reconstruction is filtered back-projection algorithm [46, 47]. This algorithm filters the images and then back-projects them to the 3-D image volume [10].

In the image enhancement process, smoothing filters, e.g. Box filters and Gauss filters [47, 48], are usually applied to images to eliminate the influence of noise. Median filters are also used when there are outliers in the images. All the filters listed above have low-pass characteristics and are spatially invariant [10]. The smoothing filters have negative effect on the sharp edges in the image. Research has been carried out to find a low-pass filter which suppresses the noise without sacrificing the edge sharpness. The solutions involve numerically solving partial differential equations (PDEs). They are the non-liner diffusion filter [49, 50], the shock filter [51] and the inverse scale space filter [52]. More details on different type of filters can be found in the book written by Aubert and Kornprobst [53]. These filters were designed for 2-D image processing. Since 3-D reconstruction is based on sequence of 2-D images, the filters described above can be applied to 3-D image processing.

Compared to the image enhancement method, the image classification method is more material characteristic-oriented. It highly depends on the images to be processed, or saying in another way, the property of the material to be investigated. There is no ideal method working for every case. The generally applied classification methods include histogrambased thresholding [36, 37], region growing [54,55], iterative class property minimization $[56,57]$ and segmentation [58].

Structure clustering is the process to perform interested measurement, e.g. the grain size distribution, and area fraction, etc. one example of segmentation algorithm is the watershed algorithm, which is based on the principle of a landscape flooded by water [59].

\section{Application of image processing in porous material characterization}

As a non-destructive test method, image processing-based method can perform repeated tests of the same sample. The properties of the sample can be monitored both spatially and temporally. Furthermore, with proper imaging techniques, image processing-based method can provide 3-D scanning of the sample, to obtain an in-depth view of material. This is very useful in the area of porous materials, where the 3-D structure plays an important role to the functioning. The major image processing techniques involved in characterizing porous materials include noise reduction, image enhancement, image subtraction, pixel classification and pixel clustering. 


\subsection{Porous material}

Porous material is a material with irregular pores (or voids) and solid skeletons. One simple example is the sponge. Many materials are considered as porous materials, such as rocks, soil, wood, paper, catalysts, biological tissues (bone), cements, and some ceramics. Some important properties of these materials can only be rationalized when they are treated as porous media. The application area of porous materials is so wide that it includes from rock mechanics, petroleum engineering, to filtration, acoustics, biology and material science. The featured properties of porous materials include density, porosity, pore size, permeability and mechanical strength. The structure of porous materials has been modeled in three different ways - networks of capillaries, arrays of solid particles and trimodal.

The complicated structures of porous materials bring significant challenge to characterization techniques. Owing to the aspect of non-destructive and deep investigating capability, image processing-based techniques have been applied to characterize porous materials in many different ways. The properties can be detected include the 2-D properties of porosity [60], pore diameter [60,61] and 3-D structure [62-64], as well as flow behavior inside the porous media [10].

\subsection{Surface porosity and pore diameter}

Surface porosity and pore diameter are the fundamental properties of porous materials. The detection of these two properties starts with imaging techniques to differentiate the pores from the frame of the porous materials. One way to do that is filling the pores with resins. After that the sample will be cut and polished for subsequent imaging [65]. The spatial resolution of this technique not only depends on the imaging hardware, but also the image processing algorithm. Due to the limitation of image segmentation and mathematic morphology filters, the pores can only be detected when they span more than five pixels. Prado et al obtained a resolution of $50 \mu \mathrm{m}$ using this method [65].

Wang et al. investigated the surface porosity of a sintered porous material using a different imaging technique [60]. The penetrating pores were indicated by water coming out from the bottom through them. Images of the top surface were taken before and after the water reaching the top surface but not flowing out. In this process, all the test conditions remained the same except for the water flow. In these two images, the brightness of the pixels at the place where the penetrating pores locate was different, because the penetrating pores reflect more light when filled by water. Image subtraction was applied to these images subsequently, resulting in an image with the information of the penetrating pores. The porosity and pore diameter can be measured by analyzing the subtracted image, using thresholding, edge detection, etc. The typical images are shown in Fig. 1. The usage of water flooding and visible light illumination make the detection of penetrating pores safe and convenient. The image subtraction method applied here eliminated the influence of hardware noise, e.g. dark current noise and pixel non-uniformity noise. The optical measurement and image processing showed their property of non-destructive here. However, limited by the imaging technique, this measurement only applies to specimens with a flat surface. 


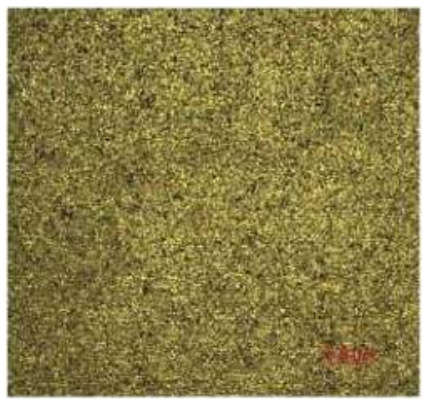

(a)

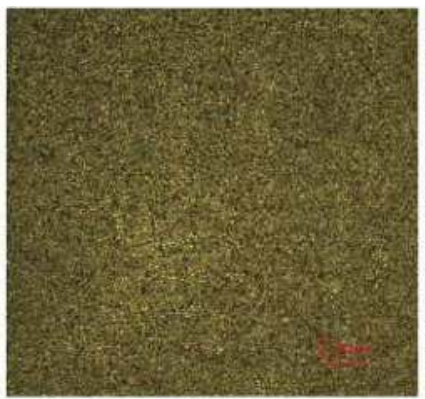

(b)

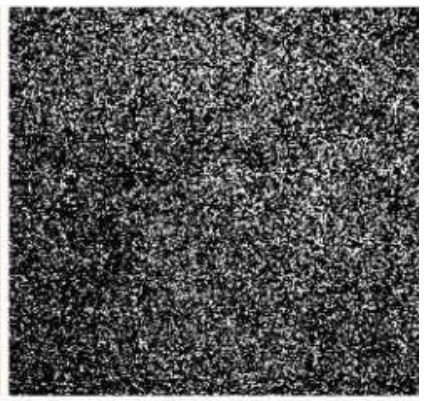

(c)

Fig. 1. (a) Digital camera image of the porous surface; open pores fully filled with water but not to overflowing. (b) Water level has receded from the penetrating pores. (c) Binary image containing information of the penetrating pores; white pixels indicate pores [60].

\subsection{3-D structure}

3-D structures of porous materials and fluid flow inside them have attracted more and more interest recently. For example, in the porous materials used for transpiration cooling, the distribution of the penetrating pores and how the coolant flows through them significantly affect the cooling efficiency of this material. The protocol to obtain 3-D geometric information of porous material is based on stacking 2-D image slices. There are several imaging techniques available to obtain these 2-D images and scan in the third dimension. One major imaging technique is computed tomography (CT). Based on the facility used, it can be further categorized to X-ray-based and neutron-based method [10]. These two methods are based on observing the scattered intensity of X-ray and neutron, which represents the difference in density, polarization, or scattered angle of the sample. The resolution of the X-ray microtomography can be as low as $0.1 \mu \mathrm{m}$. Another imaging technique is Magnetic Resonance Imaging (MRI) [66], which has the resolution of tens of nanometers. For even higher resolution, a dual-beam Focused Ion Beam (FIB) imaging method has been proposed to investigate the 3-D geometry of porous materials [67]. The voxel resolution of this method is $15 \mathrm{~nm}$.

The examples of 3-D geometric investigation of porous materials range from soil to biological tissues. A. Kaestner et al. measured the hydraulic properties of soil aggregate packing using both neutron radiography and X-ray tomography [10]. The neutron radiography has the ability to show water distribution within a sample, while $X$-ray tomography shows the structures at higher resolution. L. Pothuaud et al. investigated the microarchitecture of trabecular bone using MRI [66]. In the image processing part, they interpreted the graphs in terms of vertices and branches. A six-connection algorithm was applied to form 3-D structure. Fig. 2 shows the procedure of 3-D reconstruction of an $\mathrm{Al}$ foam using 2-D images [68]. The Al foam was sectioned physically, and then images of each slice were taken consequently. However, the image processing technique remained the same as that for other 3-D imaging methods, e.g. MRI.

More research work on 3-D reconstitution of porous media from image processing can be found in the area of soil [69-74], ceramics [75, 76], polyethylene [77], bone [78], metal [79, 80] and general porous media [81, 82]. 

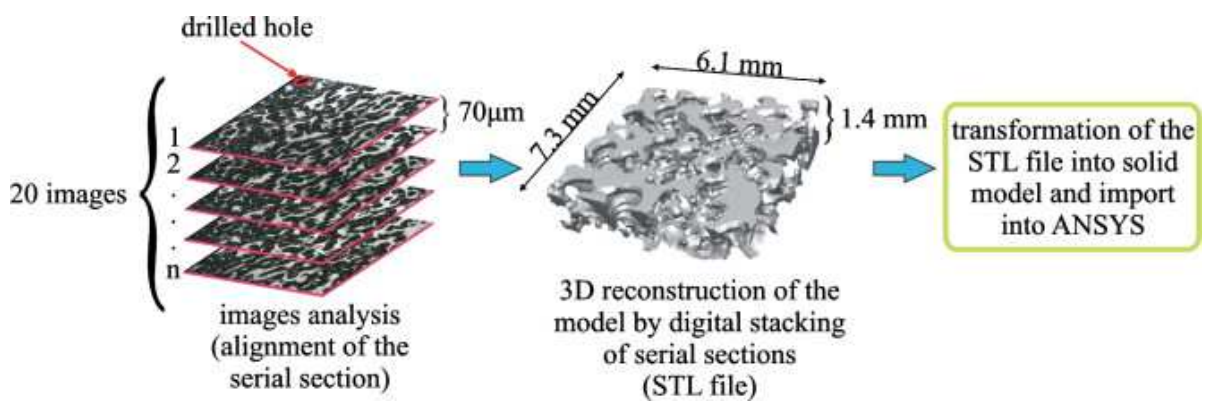

$3 \mathrm{D}$ reconstruction of the

model by digital stacking of serial sections

(STL file)

Fig. 2. Schematic diagram accounting for the image processing to obtain the 3D model of the Al-foam [68].

\subsection{Fluid behavior}

As discussed before, fluid behavior inside the porous material has significant effect on its performance. Not only for the sintered porous media used for transpiration cooling, but also for the porous material used as catalyst carrier, the fluid flow and diffusion inside the pore network is doomed to be important. Simulation work of fluid flow in a porous medium has been conducted for decades, which usually involves solving transportation equations for an unsteady state. Speaking of the experimental work, during the last two decades, the nuclear magnetic resonance (NMR) technique has been proven to be a successful imaging technique [83]. This technique measures the Fourier transform of self-diffusion propagator of the inside fluid [84]. Fig. 3 shows multiphase fluid core saturation through sodium NMR signal measurement [85]. In the mixture of water and oil, sodium only present in the water phase. Therefore, those two fluid can be distinguished by imaging the sodium component. This research has application in the area of rock content analysis and petroleum industry.
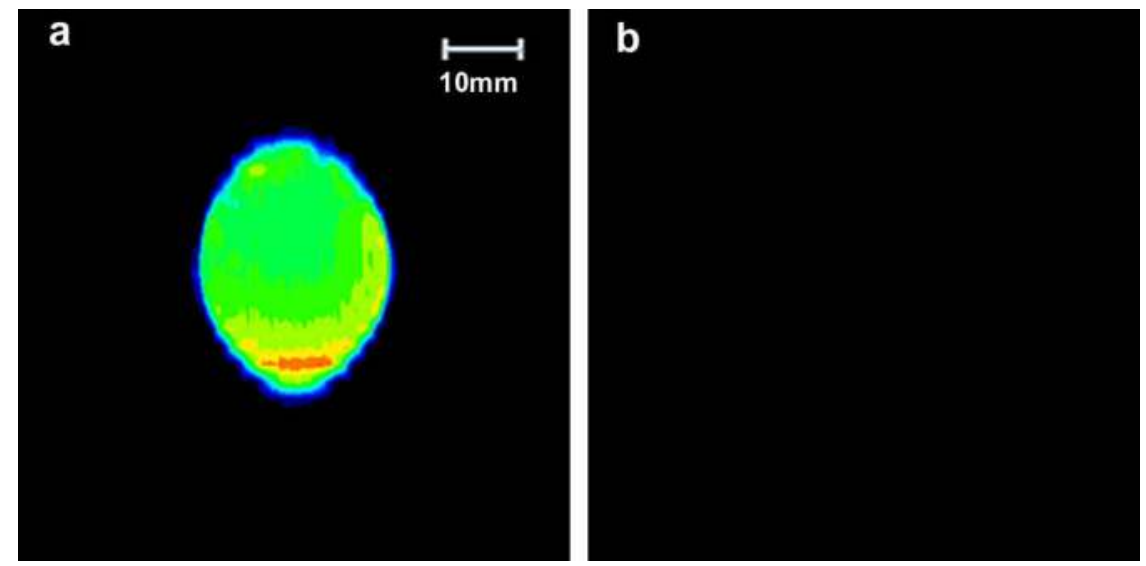

Fig. 3. 2D axial images of a Bentheimer core (a) fully saturated with $100 \mathrm{kppm}$ brine (b) fully saturated with Isopar L [85].

Research work on fluid behavior in porous material has also been performed to the material of polyethylene [77], rocks [86-88] and general porous materials [87, 89]. 


\section{Conclusion}

In conclusion, image processing-based measurement techniques have the advantages of being non-destructive, easy operation, in-depth 3-D view capability and considerably high resolution. It has wide applications in material characterization area, especially the area of porous materials. The application area can be further extended with proper imaging technique. The image processing techniques involved include image alignment, image stitching, contrast enhancement, thresholding, image substraction, and 3-D reconstruction etc. Corresponding image processing techniques to be applied are highly affected by the properties of materials to be measured. The image processing methods can be migrated to other areas with similar image characteristics. For achieving accurate results, the image processing-based measurement should be improved from the image acquisition hardware, experimental setup and image processing algorithms aspects of view.

\section{References}

[1] W. S. Boyle and G. Smith, Charge-coupled semiconductor devices. Bell System Techanics of Journal, 1970. 49: p. 587-593.

[2] E. R. Fossum, CMOS image sensors: Electronic camera-on-a-chip. IEEE Transactions on Electron Devices, 1997. 44(10): p. 1689-1698.

[3] Stephen Kempainen, CMOS image sensors: Eclipsing CCDs in visual information? EDN, 1997. 42(21): p. 101-102.

[4] J. Zarnowski, M. Pace, and M. Joyner, Active-pixel CMOS sensors improve their image. Laser Focus World, 1999. 35(7): p. 111-114.

[5] Zurich Nicolas Blanc, CCD versus CMOS - has CCD imaging come to an end?, in Photogrammetic Week 01. 2001: Wichmann Verlag, Heidelberg.

[6] M. Bigas, E. Cabruja, J. Forest, and J. Salvi, Review of CMOS image sensors. Microelectronics Journal, 2006. 37(5): p. 433-451.

[7] R. Widenhorn, M. M. Blouke, A. Weber, A. Rest, and E. Bodegom, Temperature dependence of dark current in a CCD, in Sensors and Camera Systems for Scientific, Industrial, and Digital Photography Applications III. 2002, Spie-Int Soc Optical Engineering: Bellingham. p. 193-201.

[8] K. Miyaguchi, H. Suzuki, J. Dezaki, and K. Yamamoto, CCD developed for scientific application by Hamamatsu. Nuclear Instruments and Methods in Physics Research Section A: Accelerators, Spectrometers, Detectors and Associated Equipment, 1999. 436(1-2): p. 24-31.

[9] G. E. Healey and R. Kondepudy, Radiometric CCD camera calibration and noise estimation. IEEE Transactions on Pattern Analysis and Machine Intelligence, 1994. 16(3): p. 267276.

[10] A. Kaestner, E. Lehmann, and M. Stampanoni, Imaging and image processing in porous media research. Advances in Water Resources, 2008. 31(9): p. 1174-1187.

[11] Michael J. Black and Anand Rangarajan, On the unification of line processes, outlier rejection, and robust statistics with applications in early vision. Int. J. Comput. Vision, 1996. 19(1): p. 57-91.

[12] M. J. Hannah, Computer Matching of Areas in Stereo Images. 1974, Stanford University.

[13] M. J. Hannah, Test results from SRI's stereo system, in Image Understanding Workshop. 1988, Morgan Kaufmann Publishers: Cambridge, Massachusetts. p. 740-744.

[14] H. P. Moravec, The Stanford Cart and the CMU Rover. Proceedings of the IEEE, 1983. 71(7): p. 872-884. 
[15] D. S. Zhang and G. J. Lu, A comparative study of curvature scale space and Fourier descriptors for shape-based image retrieval. Journal of Visual Communication and Image Representation, 2003. 14(1): p. 41-60.

[16] Iivari Kunttu, Leena Lepisto, Juhani Rauhamaa, and Ari Visa, Fourier-based object description in defect image retrieval. Machine Vision and Applications, 2006. 17(4): p. 211-218.

[17] Bruce D. Lucas and Takeo Kanade, An iterative image registration technique with an application to stereo vision, in Proceedings of the 7th international joint conference on Artificial intelligence - Volume 2. 1981, Morgan Kaufmann Publishers Inc.: Vancouver, BC, Canada. p. 674-679.

[18] Qi Tian and Michael N. Huhns, Algorithms for subpixel registration. Computer Vision, Graphics, and Image Processing, 1986. 35(2): p. 220-233.

[19] W. Forstner, A feature-based correspondence algorithm for image matching. . Intl. Arch. Photogrammetry \& Remote Sensing, 1986. 26(3): p. 150-166.

[20] C. Harris and M. Stephens. A Combined Corner and Edge Detection. in Proceedings of The Fourth Alvey Vision Conference. 1988.

[21] Jianbo Shi and Tomasi. Good features to track. in Computer Vision and Pattern Recognition, 1994. Proceedings CVPR '94., 1994 IEEE Computer Society Conference on. 1994. Seattle, WA, USA.

[22] Richard Szeliski, Video Mosaics for Virtual Environments. IEEE Comput. Graph. Appl., 1996. 16(2): p. 22-30.

[23] Richard Szeliski and Heung-Yeung Shum. Creating full view panoramic image mosaics and texture-mapped models. in Computer Graphics. 1997. SIGGRAPH'97, Los Angeles: Association for Computing Machinery, Inc.

[24] H. Y. Shum and R. Szeliski, Construction of panoramic image mosaics with global and local alignment (vol 36, pg 101, 2000). International Journal of Computer Vision, 2002. 48(2): p. 151-152.

[25] Satyan Coorg and Seth Teller, Spherical Mosaics with Quaternions and Dense Correlation. Int. J. Comput. Vision, 2000. 37(3): p. 259-273.

[26] Richard Szeliski, Image Alignment and Stitching: A Tutorial. 2006, Microsoft Corporation.

[27] Stephen M. Pizer, E. Philip Amburn, John D. Austin, Robert Cromartie, Ari Geselowitz, Trey Greer, Bart ter Haar Romeny, John B. Zimmerman, and Karel Zuiderveld, Adaptive histogram equalization and its variations. Computer Vision, Graphics, and Image Processing, 1987. 39(3): p. 355-368.

[28] Tae Kim, Joon Paik, and Bong Kang, Contrast enhancement system using spatially adaptive histogram equalization with temporal filtering. IEEE Transactions on Consumer Electronics, 1998. 44(1): p. 82-87.

[29] D. Coltuc, P. Bolon, and J. M. Chassery, Exact histogram specification. IEEE Transactions on Image Processing, 2006. 15(5): p. 1143-1152.

[30] J. L. Starck, F. Murtagh, E. J. Candes, and D. L. Donoho, Gray and color image contrast enhancement by the curvelet transform. IEEE Transactions on Image Processing on, 2003. 12(6): p. 706-717.

[31] S. Dippel, M. Stahl, R. Wiemker, and T. Blaffert, Multiscale contrast enhancement for radiographies: Laplacian pyramid versus fast wavelet transform. IEEE Transactions on Medical Imaging, 2002. 21(4): p. 343-353.

[32] Wan Yi and Shi Dongbin, Joint Exact Histogram Specification and Image Enhancement Through the Wavelet Transform. IEEE Transactions on Image Processing, 2007. 16(9): p. 2245-2250. 
[33] D. T. Cobra, Image histogram modification based on a new model of the visual system nonlinearity. Journal of Electronic Imaging, 1998. 7(4): p. 807-815.

[34] H. Liu and C. F. Nodine, Generalized image contrast enhancement technique based on the Heinemann contrast discrimination model. Journal of Electronic Imaging, 1996. 5(3): p. 388-395.

[35] Chang Yuchou, Lee Dah-Jye, J. Archibald, and Hong Yi. Using collaborative learning for image contrast enhancement. in Pattern Recognition, 2008. ICPR 2008. 19th International Conference on. 2008.

[36] N. Otsu, A threshold selection method from gray-level histograms. IEEE Transactions on Systems, Man and Cybernetics, 1979. 9(1): p. 62-66.

[37] Steven M. Kay, Fundamentals of Statistical Signal Processing, Volume 2: Detection Theory. 1998: Prentice Hall.

[38] J.N. Kapur, P.K. Sahoo, and A.K.C. Wong, A new method for gray-level picture thresholding using the entropy of the histogram. Computer Vision, Graphics, and Image Processing, 1985: p. 273-285.

[39] S. Hemachander, A. Verma, S. Arora, and Prasanta K. Panigrahi, Locally adaptive block thresholding method with continuity constraint. Pattern Recognition Letters, 2007. 28(1): p. 119-124.

[40] J. M. White and G. D. Rohrer, Image thresholding for optical character-recognition and other applications requiring character image extraction. IBM Journal of Research and Development, 1983. 27(4): p. 400-411.

[41] K. V. Mardia and T. J. Hainsworth, A Spatial Thresholding Method for Image Segmentation. IEEE Trans. Pattern Anal. Mach. Intell., 1988. 10(6): p. 919-927.

[42] C. H. Li and C. K. Lee, Minimum cross entropy thresholding. Pattern Recognition, 1993. 26(4): p. 617-625.

[43] Jamal Saeedi, Mohammad Hassan Moradi, and Karim Faez, A new wavelet-based fuzzy single and multi-channel image denoising. Image and Vision Computing, 2010. 28(12): p. 1611-1623.

[44] Shitong Wang, Fu-lai Chung, and Fusong Xiong, A novel image thresholding method based on Parzen window estimate. Pattern Recognition, 2008. 41(1): p. 117-129.

[45] M. Sezgin and B. Sankur, Survey over image thresholding techniques and quantitative performance evaluation. Journal of Electronic Imaging, 2004. 13(1): p. 146-168.

[46] Avinash C. Kak and Malcolm Slaney, Principles of Computerized Tomographic Imaging. 1988: IEEE Press.

[47] Anil K. Jain, Fundamentals of Digital Image Processing. 1988: Prentice Hall.

[48] Jahne B., Digital Image Processing. 2 ed. 2002: Springer-Verlag.

[49] P. Perona and J. Malik, Scale-space and edge detection using anisotropic diffusion. IEEE Transactions on Pattern Analysis and Machine Intelligence, 1990. 12(7): p. 629-639.

[50] F. Catte, P. L. Lions, J. M. Morel, and T. Coll, Image selective smoothing and edge detection by nonlinear diffusion. SIAM J. Numer. Anal., 1992. 29(1): p. 182-193.

[51] Stanley Osher and Leonid Rudin, Feature-oriented image enhancement using shock filters. SIAM J. Numer. Anal., 1990. 27(4): p. 919-940.

[52] L. I. Rudin, S. Osher, and E. Fatemi, Nonlinear total variation based noise removal algorithms. Physica D, 1992. 60(1-4): p. 259-268.

[53] Gilles Aubert and Pierre Kornprobst, Mathematical problems in image processing: Partial Differential Equations and the Calculus of Variations. , in Applied Mathematical Sciences. 2002, Springer-Verlag.

[54] R. Adams and L. Bischof, Seeded region growing. IEEE Transactions on Pattern Analysis and Machine Intelligence, 1994. 16(6): p. 641-647. 
[55] Jianping Fan, Guihua Zeng, Mathurin Body, and Mohand-Said Hacid, Seeded region growing: an extensive and comparative study. Pattern Recognition Letters, 2005. 26(8): p. 1139-1156.

[56] Wonho Oh and W. Brent Lindquist, Image Thresholding by Indicator Kriging. IEEE Trans. Pattern Anal. Mach. Intell., 1999. 21(7): p. 590-602.

[57] Riyadh I. Al-Raoush and Clinton S. Willson, A pore-scale investigation of a multiphase porous media system. Journal of Contaminant Hydrology, 2005. 77(1-2): p. 67-89.

[58] Peter J. Burt, Tsai-Hong Hong, and Azriel Rosenfeld, Segmentation and Estimation of Image Region Properties through Cooperative Hierarchial Computation. IEEE Transactions on Systems, Man and Cybernetics, 1981. 11(12): p. 802-809.

[59] Pierre Soille, Morphological Image Analysis: Principles and Applications. 2 ed. 2004: Springer.

[60] Jianhua Wang, Ming Gan, and Junxiang Shi, Detection and characterization of penetrating pores in porous materials. Materials Characterization, 2007. 58(1): p. 8-12.

[61] F. H. She, K. L. Tung, and L. X. Kong, Calculation of effective pore diameters in porous filtration membranes with image analysis. Robotics and Computer-Integrated Manufacturing, 2008. 24(3): p. 427-434.

[62] J. F. Daian, C. P. Fernandes, P. C. Philippi, and J. A. Bellini da Cunha Neto, 3D reconstitution of porous media from image processing data using a multiscale percolation system. Journal of Petroleum Science and Engineering, 2004. 42(1): p. 15-28.

[63] S. Blacher, V. Maquet, F. Schils, D. Martin, J. Schoenen, G. Moonen, R. Jerome, and J. P. Pirard, Image analysis of the axonal ingrowth into poly(,-lactide) porous scaffolds in relation to the 3-D porous structure. Biomaterials, 2003. 24(6): p. 1033-1040.

[64] Elena Sevostianova, Bernd Leinauer, and Igor Sevostianov, Quantitative characterization of the microstructure of a porous material in the context of tortuosity. International Journal of Engineering Science, 2010. 48(12): p. 1693-1701.

[65] B. Prado, C. Duwig, J. Marquez, P. Delmas, P. Morales, J. James, and J. Etchevers, Image processing-based study of soil porosity and its effect on water movement through Andosol intact columns. Agricultural Water Management, 2009. 96(10): p. 1377-1386.

[66] L. Pothuaud, P. Porion, E. Lespessailles, C. L. Benhamou, and P. Levitz, A new method for three-dimensional skeleton graph analysis of porous media: application to trabecular bone microarchitecture. Journal of Microscopy, 2000. 199(2): p. 149-161.

[67] L. Holzer, F. Indutnyi, P. H. Gasser, B. MÜNch, and M. Wegmann, Three-dimensional analysis of porous BaTiO3 ceramics using FIB nanotomography. Journal of Microscopy, 2004. 216(1): p. 84-95.

[68] N. Michailidis, F. Stergioudi, H. Omar, and D. N. Tsipas, An image-based reconstruction of the $3 D$ geometry of an $\mathrm{Al}$ open-cell foam and FEM modeling of the material response. Mechanics of Materials, 2010. 42(2): p. 142-147.

[69] J. F. Daïan, C. P. Fernandes, P. C. Philippi, and J. A. Bellini da Cunha Neto, 3D reconstitution of porous media from image processing data using a multiscale percolation system. Journal of Petroleum Science and Engineering, 2004. 42(1): p. 15-28.

[70] Erwan Plougonven and Dominique Bernard, Optimal removal of topological artefacts in microtomographic images of porous materials. Advances in Water Resources, 2011. 34(6): p. 731-736.

[71] Riyadh Al-Raoush and Khalid A. Alshibli, Distribution of local void ratio in porous media systems from 3D X-ray microtomography images. Physica A: Statistical Mechanics and its Applications, 2006. 361(2): p. 441-456.

[72] Olivier Monga, Defining and computing stable representations of volume shapes from discrete trace using volume primitives: Application to $3 D$ image analysis in soil science. Image and Vision Computing, 2007. 25(7): p. 1134-1153. 
[73] W. Wang, A. N. Kravchenko, A. J. M. Smucker, and M. L. Rivers, Comparison of image segmentation methods in simulated $2 D$ and $3 D$ microtomographic images of soil aggregates. Geoderma, 2011. 162(3-4): p. 231-241.

[74] Jean-François Delerue and Edith Perrier, DXSoil, a library for 3D image analysis in soil science. Computers \& Geosciences, 2002. 28(9): p. 1041-1050.

[75] Eric Maire, Paolo Colombo, Jerome Adrien, Laurent Babout, and Lisa Biasetto, Characterization of the morphology of cellular ceramics by 3D image processing of X-ray tomography. Journal of the European Ceramic Society, 2007. 27(4): p. 1973-1981.

[76] T. Wiederkehr, B. Klusemann, D. Gies, H. Müller, and B. Svendsen, An image morphing method for $3 D$ reconstruction and FE-analysis of pore networks in thermal spray coatings. Computational Materials Science, 2010. 47(4): p. 881-889.

[77] M. Prodanovic, W. B. Lindquist, and R. S. Seright, Porous structure and fluid partitioning in polyethylene cores from $3 D$ X-ray microtomographic imaging. Journal of Colloid and Interface Science, 2006. 298(1): p. 282-297.

[78] Eduard Vergés, Dolors Ayala, Sergi Grau, and Dani Tost, 3D reconstruction and quantification of porous structures. Computers \& Graphics, 2008. 32(4): p. 438-444.

[79] Bungo Otsuki, Mitsuru Takemoto, Shunsuke Fujibayashi, Masashi Neo, Tadashi Kokubo, and Takashi Nakamura, Pore throat size and connectivity determine bone and tissue ingrowth into porous implants: Three-dimensional micro-CT based structural analyses of porous bioactive titanium implants. Biomaterials, 2006. 27(35): p. 5892-5900.

[80] T. Kujime, M. Tane, S. K. Hyun, and H. Nakajima, Three-dimensional image-based modeling of lotus-type porous carbon steel and simulation of its mechanical behavior by finite element method. Materials Science and Engineering: A, 2007. 460-461: p. 220-226.

[81] Z. R. Liang, C. P. Fernandes, F. S. Magnani, and P. C. Philippi, A reconstruction technique for three-dimensional porous media using image analysis and Fourier transforms. Journal of Petroleum Science and Engineering, 1998. 21(3-4): p. 273-283.

[82] J. T. Fredrich, 3D imaging of porous media using laser scanning confocal microscopy with application to microscale transport processes. Physics and Chemistry of the Earth, Part A: Solid Earth and Geodesy, 1999. 24(7): p. 551-561.

[83] P. T. Callaghan, A. Coy, D. MacGowan, K. J. Packer, and F. O. Zelaya, Diffraction-like effects in NMR diffusion studies of fluids in porous solids. Nature, 1991. 351(6326): p. 467-469.

[84] P. Levitz, Toolbox for 3D imaging and modeling of porous media: Relationship with transport properties. Cement and Concrete Research, 2007. 37(3): p. 351-359.

[85] Kathryn E. Washburn and Guillaume Madelin, Imaging of multiphase fluid saturation within a porous material via sodium NMR. Journal of Magnetic Resonance. 202(1): p. 122-126.

[86] S. Galaup, R. Burlot, A. Cerepi, L. Wang, and M. Dai, Modelisation and circulation of fluids in geological porous systems. Images analyzing and mercury porosimetry, in Studies in Surface Science and Catalysis, F.R.-R.J.R. P.L. Llewellyn and N. Seaton, Editors. 2007, Elsevier. p. 705-712.

[87] Yannick Anguy, Robert Ehrlich, Azita Ahmadi, and Michel Quintard, On the ability of a class of random models to portray the structural features of real, observed, porous media in relation to fluid flow. Cement and Concrete Composites, 2001. 23(2-3): p. 313-330.

[88] Tetsuro Hirono, Manabu Takahashi, and Satoru Nakashima, In situ visualization of fluid flow image within deformed rock by X-ray CT. Engineering Geology, 2003. 70(1-2): p. 37-46.

[89] Nejib Smaoui and Ridha B. Gharbi, Using Karhunen-Loéve decomposition and artificial neural network to model miscible fluid displacement in porous media. Applied Mathematical Modelling, 2000. 24(8-9): p. 657-675. 


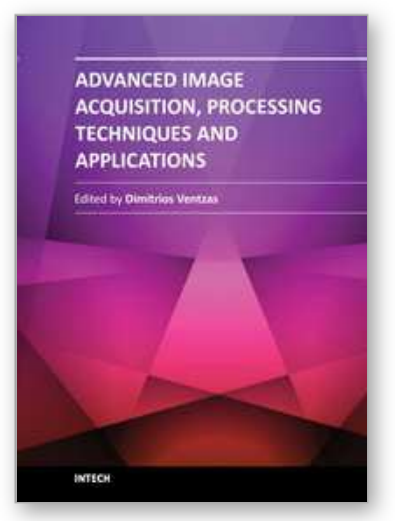

\author{
Advanced Image Acquisition, Processing Techniques and \\ Applications I \\ Edited by Dr. Dimitrios Ventzas
}

ISBN 978-953-51-0342-4

Hard cover, 170 pages

Publisher InTech

Published online 14, March, 2012

Published in print edition March, 2012

"Advanced Image Acquisition, Processing Techniques and Applications" is the first book of a series that provides image processing principles and practical software implementation on a broad range of applications. The book integrates material from leading researchers on Applied Digital Image Acquisition and Processing. An important feature of the book is its emphasis on software tools and scientific computing in order to enhance results and arrive at problem solution.

\title{
How to reference
}

In order to correctly reference this scholarly work, feel free to copy and paste the following:

Ming Gan and Jianhua Wang (2012). Applications of Image Processing Technique in Porous Material Characterization, Advanced Image Acquisition, Processing Techniques and Applications I, Dr. Dimitrios Ventzas (Ed.), ISBN: 978-953-51-0342-4, InTech, Available from: http://www.intechopen.com/books/advancedimage-acquisition-processing-techniques-and-applications-i/applications-of-image-processing-technique-inporous-material-characterization

\section{INTECH}

open science | open minds

\section{InTech Europe}

University Campus STeP Ri

Slavka Krautzeka 83/A

51000 Rijeka, Croatia

Phone: +385 (51) 770447

Fax: +385 (51) 686166

www.intechopen.com

\section{InTech China}

Unit 405, Office Block, Hotel Equatorial Shanghai

No.65, Yan An Road (West), Shanghai, 200040, China

中国上海市延安西路 65 号上海国际贵都大饭店办公楼 405 单元

Phone: +86-21-62489820

Fax: +86-21-62489821 
(C) 2012 The Author(s). Licensee IntechOpen. This is an open access article distributed under the terms of the Creative Commons Attribution 3.0 License, which permits unrestricted use, distribution, and reproduction in any medium, provided the original work is properly cited. 